\title{
Rasgos de personalidad, percepción de apoyo social y motivación de logro como predictores del rendimiento académico en estudiantes pertenecientes a programas de inclusión y permanencia en la educación universitaria (PACE)
}

Traços de personalidade, percepção de apoio social e motivação de desempenho como preditores de desempenho acadêmico em estudantes pertencentes a programas de inclusão e permanência no ensino universitário (PACE)

Personality traits, perception of social support and motivation of achievement as predictors of academic performance in students belonging to programs of inclusion and permanence in university education (PACE)

\author{
Mario Morales Navarro \\ Universidad de Santiago de Chile \\ Eduardo Guzmán Utreras (iD) \\ Universidad Santo Tomás, Chile \\ Carmen Gloria Baeza Ugarte (iD \\ Universidad de Santiago de Chile
}

RESUMEN En este trabajo se presentan los resultados de una investigación cuyo objetivo fue estudiar de qué manera ciertas variables personales (rasgos de personalidad, percepción de apoyo social y motivación de logro) de los estudiantes pueden explicar su rendimiento académico, específicamente en estudiantes pertenecientes a un programa de acompañamiento para el acceso a la educación superior (PACE). Se contó con una muestra no probabilística e incidental de 155 estudiantes de una universidad pública durante el primer semestre. Los resultados muestran que no existen diferencias significativas entre hombres y mujeres en cuanto a las variables estudiadas. Además, se evidenciaron correlaciones significativas entre el rendimiento académico y la motivación de logro, apoyo social de la red cercana (amigos), la percepción de apoyo familiar, y con el 
rasgo de personalidad apertura a la experiencia. Por medio de una regresión lineal múltiple, se obtuvo que las variables personales de este estudio son capaces de explicar un $33,3 \%$ de la varianza en rendimiento académico. Se plantea la necesidad de profundizar acerca de estos factores que se asocian a la permanencia universitaria de los estudiantes provenientes de sectores vulnerables.

PALABRAS CLAVE PACE, motivación, personalidad, apoyo social, rendimiento académico.

RESUMO Os resultados de uma investigação cujo objetivo foi estudar como determinadas variáveis pessoais (traços de personalidade, percepção de apoio social e motivação para a realização) de alunos podem explicar o desempenho acadêmico, especificamente em estudantes pertencentes a um programa de acompanhamento para alunos. acesso ao ensino superior (PACE). Houve uma amostra não probabilística e incidental de 155 estudantes universitários de uma universidade pública durante o primeiro semestre. Os resultados mostram que não há diferenças significativas entre homens e mulheres em relação às variáveis estudadas. Além disso, houve correlações significativas entre desempenho acadêmico e motivação para a conquista, apoio social da rede vizinha (amigos), percepção do apoio familiar e o traço de personalidade aberto à experiência. Por meio de regressão linear múltipla, obteve-se que as variáveis pessoais deste estudo são capazes de explicar 33,3\% da variação no desempenho acadêmico. Surge a necessidade de aprofundar esses fatores associados à permanência universitária de estudantes de setores vulneráveis.

PALAVRAS-CHAVE PACE, Motivação, personalidade, universidade, vulnerabilidade, ritmo.

ABSTRACT We present the results of an investigation whose objective was to study how certain personal variables (personality traits, perception of social support and motivation for achievement) of students can explain the academic performance, specifically in students belonging to an accompaniment program for access to higher education (PACE). There was a non-probabilistic and incidental sample of 155 university students from a public university during the first semester. The results show that there are no significant differences between men and women regarding the variables studied. In addition, there were significant correlations between academic performance and motivation for achievement, social support from the nearby network (friends), the perception of family support, and with the personality trait open to experience. Through a multiple linear regression, it was obtained that the personal variables of this study can explain $33.3 \%$ of the variance in academic performance. The need arises to deepen about these factors that are associated with the university permanence of students from vulnerable sectors.

KEYWORDS PACE, Motivation, personality, social support, academic performance. 


\section{Introducción}

El caso de Chile probablemente sea uno de los ejemplos más significativos en materia de segregación y desigualdad educacional. Ya en 1930 se instaló un proceso único de selección y admisión obligatorio sobre la hipótesis de que no todos los estudiantes tenían las habilidades, condiciones personales ni académicas para continuar estudios considerados como superiores, lo que operó en forma constante hasta 1981 cuando el surgimiento de las universidades privadas rompió esta hegemonía (Donoso y Schiefelbein, 2007: 9). No obstante, los estudiantes que han desarrollado su escolaridad en sectores considerados como educacional y socialmente vulnerables han observado con distancia cómo la educación universitaria amplía su cobertura y oferta académica, sin brindar oportunidades que reivindiquen su situación de desventaja social. Pese a que una serie de políticas públicas y privadas asociadas al otorgamiento de becas al mérito académico financiaron en parte o en su totalidad a este tipo de estudiantes, el problema en el acceso a este nivel de educación continuó siendo la vulneración académica escolar asociada a los déficits en el cumplimiento curricular, por una parte, y a los grandes problemas pedagógicos por otro, donde los principales esfuerzos se han orientado a la reproducción lineal de información más que al desarrollo del pensamiento y los potenciales personales, por tanto, más a la mera obtención de resultados que permitieran egresar de la enseñanza media-secundaria y no a la posibilidad de continuar estudios de nivel avanzado o universitario (Toirkens y Osses, 2016).

En el ámbito primario y secundario las grandes brechas internas se han mantenido, reproduciendo a lo largo de los años diferencias principalmente atribuidas a una condición sociocultural donde se insertan las instituciones de educación. Esto se ha mantenido, en primer lugar a pesar de que en la última década la contingencia educacional y las diversas demandas sociales latinoamericanas han puesto sobre la mesa la importancia de discutir acerca de la educación formal como un espacio de derecho, donde se garanticen la calidad y la igualdad social, respondiendo a una concepción regional que asume la importancia de la educación como mecanismo para reducir la desigualdad social, a partir del desarrollo y de la asunción de las familias (Aequalis, 2013); y en segundo lugar, a pesar, de que en el ámbito terciario en las últimas dos décadas se ha abandonado la realidad elitista y homogénea debido al aumento de la oferta académica de instituciones universitarias.

Este continuo de progresión entre educación primaria y secundaria va construyendo una trayectoria educacional con una serie de déficits académicos en desmedro de un rendimiento exitoso que a futuro permita una libre elección de los y las estudiantes frente al dilema trabajo/continuidad de estudios, lo que al mismo tiempo es percibido como una grave situación que no ha sido debidamente atendida por los estamentos de Estado, produciéndose una merma en las oportunidades de acceso a las distintas universidades o instituciones terciarias. 
Como forma de aminorar estas desigualdades y a consecuencia de dichas demandas sociales que destacaron su prevalencia histórica, se crearon algunos mecanismos de reivindicación a estas desigualdades. Específicamente de la mano de dos universidades públicas, ${ }^{1}$ se desarrollaron una serie de programas de índole local y cultural cuyo fin fue asumir la responsabilidad social de la inclusión universitaria mediante políticas específicas que articularon el acceso a las instituciones con el reconocimiento de los talentos académicos escolares de los estudiantes, constituyéndose el primer antecedente contemporáneo ${ }^{2}$ de lo que hoy se conoce como el Programa de Acompañamiento y Acceso a la Educación Superior (PACE).

\section{Vulneración y logro educacional}

La vulnerabilidad se define hoy como un fenómeno multicausal donde convergen factores individuales y colectivos (Golovanevsky, 2007), pese a lo cual aún se mantiene su carácter polisémico. Desde una perspectiva crítica, la vulnerabilidad transita hacia lo vulnerado, no entendiéndose como un fenómeno creado donde se desconoce al sujeto portador del problema, sino como a un sujeto que es víctima de un contexto que lo vulnera arbitrariamente a través de políticas sociales y económicas regulatorias (Infante y otros, 2012). En este sentido, Nanculeo (2014) señala que la superación de una condición de vulnerabilidad/vulneración se logra mediante la movilización de las personas para resolver y solucionar condiciones sociales a las cuales no pueden acceder dependiendo de una serie de factores económicos o culturales.

Desde el punto de vista educativo, para Goldthorpe (2012) la desigualdad se explicaría por diferencias de recursos materiales y psicológicos en función de costos, beneficios sociales y posibilidades de ascenso social-familiar, produciéndose entonces los distintos esfuerzos mediante los cuales una familia no vulnerable (de clase alta) tenderá a mantener su estatus académico con su familia, a diferencia de una vulnerable (de clase baja), cuyo descenso en la escala social es imposible y no requiere dar suficiente importancia a la educación familiar (Martínez, 2014).

Diferente es la posición de Basil Bernstein, quien considera que existe una distancia entre la cultura escolar y la familia siendo más vulnerables socialmente las que deben enfrentarse a una cultura que les es ajena y desafiante, y que impone más requisitos para otorgarle un espacio, por tanto, un título educacional, consagrando así la obten-

1. Universidad de Chile en 2008 y Universidad de Santiago de Chile en 2011.

2. En 1970, la Universidad Técnica del Estado (UTE), anterior a la Universidad de Santiago de Chile (USACH), emprendió el desafío de suprimir la prueba de aptitud académica como requisito de selección a la universidad, reemplazándola por una ponderación constituida por el rendimiento académico de los últimos tres años de educación media (secundaria) (Castro, Frites y Vargas, 2016). 
ción de títulos superiores para un grupo de estudiantes privilegiados que mantienen el estatus social impuesto por las mismas clases sociales (Escudero, 2015: 15).

En Chile, la determinación de la vulnerabilidad escolar considera en primera línea factores socioeconómicos, dejando atrás aspectos psicológicos, trayectorias educativas y las experiencias socioculturales de los estudiantes, transformándolos en potenciales desertores del sistema formal (Espinoza y otros, 2012:138). Las escuelas son denominadas como vulnerables de acuerdo con el índice de vulnerabilidad escolar (IVE), entendiendo el origen de esta condición en las familias y los estudiantes a partir de una serie de criterios: el ingreso familiar; el nivel ocupacional del jefe o la jefa de hogar; el nivel de escolaridad de la madre; el hacinamiento del hogar; y la condición de ruralidad y desempeño difícil, entre otros (Ministerio de Educación, 2008).

Los planteamientos sobre la vulnerabilidad y su discusión en profundidad permiten comprender más en detalle los criterios de origen de los programas de acompañamiento y acceso a la educación superior, su política interna y la lógica cultural hacia donde apuntan en la sociedad actual, respondiendo a las principales demandas actuales en materia de inclusión y equidad en el acceso a la educación universitaria o técnica de nivel superior.

\section{El programa de acompañamiento y acceso a la educación superior}

Fue en 2014 cuando el plan piloto del denominado Programa de Acompañamiento y Acceso Efectivo a la Educación Superior inició su implementación. Este programa declara como objetivo la restitución del derecho al acceso a la educación superior de los y las estudiantes que han sido históricamente excluidos debido a barreras académicas y de formación, no pudiendo rendir en forma adecuada en las debidas evaluaciones formales de ingreso a este nivel de enseñanza, pese a que han demostrado un alto nivel de talento personal y aprovechamiento de las distintas oportunidades que su contexto les brinda. Para el cumplimiento de este objetivo, se asegura la obtención de cupos especiales que garanticen el acceso a quienes cumplan con todos los requisitos para su aprobación: posicionarse en el $15 \%$ de mejor rendimiento académico de su generación (lo que se conoce como el ranking de notas), rendir la prueba de selección universitaria (PSU), y aprobar las actividades escolares realizadas por las distintas universidades a cargo de su implementación.

En la actualidad, los programas de acompañamiento y acceso a la educación superior se han consolidado, dando cobertura a más de 84 mil estudiantes en su proceso de preparación hacia la educación superior (Mineduc, 2018). Se ha abarcado un trabajo en tres momentos del proceso de transición entre la escuela y la universidad: en la escuela (secundario); en la transición (admisión o acceso); y en la permanencia (postsecundario). A su vez, estos espacios centran sus actividades en tres grandes áreas definidas como importantes: académica, vocacional y psicológica. 
En estos tres casos se persigue la vinculación de las prácticas pedagógicas con el desarrollo de diversas competencias destacadas para el siglo XXI a las cuales se adscribe el Mineduc (2018): cognitivas, intrapersonales e interpersonales (Reimers y Chung, 2016). Centrándonos en el tercer momento -el postsecundario-, las instituciones de educación superior (IES) deben realizar una serie de actividades que busquen una articulación efectiva con el o la estudiante que accede al cupo en el programa. Estas se centran en tres hitos significativos: inducción a la vida universitaria, la que debe ser oportuna y significativa en la generación de redes de apoyo, conocimiento de la institución y participación estudiantil; diagnóstico de capacidades y necesidades académico-sociales y psicológicas de los y las estudiantes, resultando clave para implementar actividades de acompañamiento a partir de un amplio levantamiento de información (Mineduc, 2018:21); y seguimiento en el proceso de permanencia con el fin de evitar el abandono. En los tres casos se debe considerar una perspectiva transversal del estudiante.

\section{Variables personales asociadas al rendimiento académico}

Para los programas de acompañamiento y acceso a la educación superior, el rendimiento académico constituye el indicador que da cuenta del desarrollo de los talentos producto del aprovechamiento de las posibilidades contextuales que se presentan a los estudiantes (Rahmer, Miranda y Gil, 2013; Gil, Paredes y Sánchez, 2013). Este rendimiento ha sido ampliamente discutido, respaldado y criticado debido a su asociación prácticamente directa a la calidad del aprendizaje que los sujetos construyen en las instituciones formales de educación, representándose en este la apropiación de los contenidos declarados en el currículo a través de diferentes y complejos factores que actúan en ellos y ellas (Garbanzo, 2007). Constituye una medida de la capacidad de respuesta de una persona que expresa de manera estimativa lo que ha aprendido como resultado de un proceso de enseñanza (Jiménez 200o; Gaxiola y otros, 2012), constituyéndose como uno de los indicadores asociados al logro académico (De Hoyos, Espino y García, 2010). Para Edel (2003: 14), este rendimiento ha sido construido por las personas, caracterizándose por su intrincada red de articulaciones cognitivas que dan cuenta de aspectos cuantitativos y cualitativos, permitiendo la medición y predicción educativa más allá de ser tan solo un indicador de desempeño, sino permitiendo distinguir entre distintos procesos de aprendizaje y enseñanza.

\section{Personalidad y rendimiento académico en contextos universitarios}

Según Torres y Rodríguez (2006), existen una serie de variables relacionadas con el rendimiento académico, las que pueden ser internas (como las características personales del estudiante), y/o externas (que serían elementos del contexto donde se 
desenvuelve el estudiante). Dentro de dichas variables internas, se ha relacionado la teoría de los cinco factores de personalidad (Big Five) con el rendimiento académico (Niño, Calderón y Cassareto, 2003; Poropat, 2009).

O'Connor y Paunonen (2007) destacaron que los rasgos de personalidad como la responsabilidad y la apertura a la experiencia son variables fuertemente asociadas al éxito académico, asociándose esta última a un pensamiento más divergente, más creativo e imaginativo, lo que lo hace ser un predictor importante de logro a nivel intelectual (Niño, Calderón y Cassareto, 2003), mientras que la responsabilidad propicia el cumplimiento de tareas, favoreciendo además la persistencia en el logro de estas, lo que estaría íntimamente asociado a la motivación de logro personal (Cuadra y otros, 2015). Para Torres-Acosta, Rodríguez-Gómez y Acosta-Vargas (2013: 194), la mayoría de los investigadores concuerdan en que el factor de responsabilidad actúa como el mejor predictor del rendimiento académico en estudiantes universitarios, llegando a explicar hasta un $16 \%$ de la varianza, dejando de lado factores como la extraversión, que se relaciona en forma significativa con dicho rendimiento hasta los 14 y 15 años aproximadamente, tendiendo a invertirse esta relación hacia edades posteriores donde se produce la inclusión universitaria (Elliot, 1972).

Rinaudo, Barra y Donolo (2006) sostienen que a nivel universitario es importante promover el manejo por parte de los estudiantes de habilidades que favorezcan un desarrollo óptimo de patrones motivacionales caracterizados por el uso de estrategias eficaces, atribuciones adaptativas sobre sus resultados de aprendizaje y compromiso que le permita asumir un rol activo en el aprendizaje.

Desde otra perspectiva, Román y Hernández (2005) y Fernández-González, González-Hernández y Trianes-Torres (2015) afirmaron que el apoyo social percibido actúa como un factor protector que fomenta resultados académicos satisfactorios en los estudiantes. Este apoyo social se entiende como la evaluación o apreciación subjetiva que hace el estudiante del soporte que recibe por parte de sus cercanos, ya sea familia o amigos, y que lo ayudará a que se sienta comprendido y respetado dentro de su grupo. Asimismo, Torres y Rodríguez (2006) afirmaron que el apoyo social de la familia resulta importante y de gran incidencia en el rendimiento universitario, inclusive aportando en la satisfacción vital de estos estudiantes, por lo que su relevancia es significativa en este contexto (Novoa y Barra, 2015).

En resumen, el conocimiento de la incidencia de las características de personalidad, de las metas de logro académico y de la percepción de apoyo que un estudiante tenga, puede contribuir a una mejor comprensión del proceso de aprendizaje en el ambiente educativo, favoreciendo asimismo el manejo de procesos de cambios necesarios para el éxito académico. Por tanto, y sin desconocer la complejidad de la diversidad de variables que influyen en el rendimiento académico, en esta investigación se opta por trabajar con variables de orden personal como son los rasgos de personalidad, la percepción de apoyo social y la motivación de logro que presente el estudian- 
te. El rendimiento académico, al mismo tiempo, se operacionaliza en consecuencia con las principales definiciones analizadas por medio del promedio de calificaciones obtenido por los y las estudiantes

\section{Planteamiento del problema}

El objetivo de esta investigación fue obtener un modelo explicativo válido sobre el rendimiento académico en estudiantes pertenecientes a los programas de acompañamiento y acceso a la educación superior, para lo cual se procedió a identificar dicho indicador en los estudiantes (operacionalizado a través del promedio obtenido en el primer semestre lectivo), así como tres variables personales que teóricamente son convergentes y relevantes para la comprensión del fenómeno: motivación, percepción de apoyo social y rasgos de personalidad.

La determinación de estas variables surge a partir del análisis de la literatura actualmente disponible y asociada a los programas de acompañamiento y acceso a la educación superior, la que señala específicamente en su propuesta que el abordaje del diagnóstico de necesidades y capacidades personales de los estudiantes debe realizarse desde una perspectiva académica y «psicoeducativa». Cabe preguntarse acerca de cómo se realizan los procedimientos y cuáles son los factores considerados en esta perspectiva de evaluación, es decir, quién es el estudiante con el que estamos trabajando, cómo es y qué elementos de juicio se consideran para llevar adelante un programa de seguimiento personal e individual de estos estudiantes.

Distintos estudios han mostrado, con suficiencia y certeza, que aspectos como la situación familiar, la cultura escolar y la percepción de sí mismo como personaestudiante influyen significativamente en el desempeño y la permanencia académica. Desde una perspectiva individual, se ha destacado la importancia de la motivación, la valoración positiva de sí mismo, el autocontrol, la confianza en las capacidades y destrezas, y la perseverancia como factores individuales que permitirían obtener el ansiado rendimiento académico. Los estudiantes que confían en sus capacidades podrán sortear con mayores recursos los problemas que encuentren en el camino, logrando el objetivo de la titulación más allá de las adversidades del proceso y de la trayectoria (Canales y De Los Ríos, 2009). Elementos como la resiliencia, el bienestar psicológico, las expectativas docentes y personales (Morales, 2014: Morales y González 2014; Morales y Guzmán, 2016), la conformidad con la elección y apoyo en la carrera (Barahona, 2014), los déficits cognitivos asociados a las capacidades solicitadas en los contextos educativos (Jadue, 2002), y los problemas en la educación emocional (Bisquerra, 2016), son componentes que están presentes en los mecanismos personales de permanencia o abandono de estudios formales.

No obstante, y desde la perspectiva academicista actual, se aprecia en la voz de los mismos beneficiarios una tendencia hacia la consideración de aspectos meramente 
académicos como punto de partida y final en la implementación del seguimiento tutorial, preocupándose principalmente en refuerzos de tipo académicos, no dando cuenta de las necesidades individuales o sociales del sujeto-estudiante en su complejidad, lo que inclusive apunta hacia la interrupción de la trayectoria educacional exitosa que ellos y ellas han desarrollado en la secundaria (Guzmán, 2018), generando una segunda vulneración dada la transferencia emocional y de estereotipos sociales valorados como negativos más allá del contexto de origen, y que se descontextualizan a partir de estos en un lugar culturalmente distinto y adverso (Furrer, 2013:4).

En este contexto, las evidencias encontradas en la literatura (Rahmer, Miranda y Gil 2013; Gil, Paredes y Sánchez, 2013) no permiten responder con seguridad a la pregunta que nos hemos formulado en el presente estudio, la que apunta hacia quién es la persona con la que estamos trabajando en este contexto. Por una parte, es posible apreciar a un o una estudiante que proviene de un sector considerado como vulnerable a partir de una serie de determinaciones principalmente económicas y pedagógicas (índice de vulnerabilidad escolar [IVE]), y que al mismo tiempo posee ciertas expectativas con respecto a su continuidad de estudios universitarios, los que cobran un nuevo sentido al momento de instalarse el Programa de Acompañamiento y Acceso a la Educación Superior como invitación a desarrollar sus talentos personales.

En la actualidad, y considerando el reciente escenario emergente de estas políticas públicas de acceso inclusivo y permanencia o deserción universitaria de estos estudiantes, no se cuenta con estudios suficientes que profundicen acerca de los mayores desafíos con que se van enfrentando actualmente estos sujetos-estudiantes. Las distintas iniciativas de las universidades adscritas a los programas de acompañamiento y acceso a la educación superior junto con el Ministerio de Educación, han publicado estadísticas y testimonios que dan cuenta del éxito en su implementación. No obstante, aún se espera conocer en forma más amplia el proceso psicoeducativo de estos programas divididos en sus tres grandes fases: secundaria, de transición y postsecundaria, dando cuenta de una trayectoria educativa progresiva y no asimilada a un destino que amplíe las barreras que impiden la plena inclusión de los estudiantes cualquiera sea su origen, desconociendo la tan ansiada inclusión social (Dubet, 2004).

\section{Metodología}

\section{Diseño}

El estudio es cuantitativo con un diseño no experimental, de corte transversal, y utiliza una estrategia asociativa que busca la relación funcional entre las variables (Ato, López y Benavente, 2013). 


\section{Participantes}

De un universo muestral de 265 estudiantes que ingresaron a una universidad pública de Chile por medio del Programa de Acompañamiento y Acceso Efectivo de 2018, participaron de manera voluntaria 155 estudiantes, todos y todas pertenecientes activamente al primer semestre lectivo de una de las siguientes carreras profesionales: Arquitectura, Bachillerato, Derecho, Ciencias, Ciencias Médicas, Humanidades, Ingeniería, Tecnología, Química y Biología, Administración y Economía. El muestreo utilizado fue no probabilístico incidental, donde se invitó a los estudiantes a través de un correo electrónico a participar en este estudio contestando un enlace electrónico que se adjuntaba en la invitación. Del total de los participantes el $43 \%$ fueron hombres y el $57 \%$ mujeres, con una edad promedio de 18.95 años (D.S. $=0.51$ ).

Cabe destacar que el tamaño de la muestra sería adecuado para esta investigación, ya que cumpliría con la recomendación de Morales-Vallejo (2008) para estudios asociativos, el cual plantea que la muestra debe estar integrada por una cantidad de personas igual a 10 o a 20 veces más la cantidad de variables predictoras de este estudio.

\section{Instrumentos}

Para la evaluación de los rasgos de personalidad se recurrió al Big Five Inventory de John, Donahue y Kentle (1991) y se utilizó la versión en castellano adaptada de BenetMartínez y John (1998), y de Schmitt y otros (2007), con el propósito de minimizar algún inconveniente que pudiese surgir de errores de traducción. El Big Five Inventory es una escala que permite evaluar rasgos de personalidad a partir del modelo de los cinco factores. En este contexto, se invita a la persona a contestar 44 ítems entre cinco alternativas de respuesta que van desde "completamente en desacuerdo» a «completamente de acuerdo». Cada afirmación es referida a los rasgos de apertura a la experiencia, responsabilidad, extroversión, agradabilidad y neuroticismo.

Esta escala ha sido traducida a diferentes idiomas y validada en diversos países y culturas, lo que evidencia una adecuada estructura factorial y consistencia interna, la cual varía entre 0,69 y 0,79 en sus dimensiones en muestras sudamericanas (Schmitt y otros, 2007). En este estudio se calcularon los coeficientes de confiabilidad mediante un alfa de Cronbach en cada uno de los rasgos evaluados, obteniéndose medidas de confiabilidad que iban entre los 0,74 y o,82 en sus dimensiones.

La percepción de apoyo social fue medida a través de la escala AFA-R, desarrollada por González y Landero (2014). Es una escala compuesta de 14 ítems en formato escala tipo Likert de cinco alternativas de respuesta, la cual va desde nunca a siempre. Los análisis de validez dan cuenta con una estructura bifactorial destinada a evaluar la percepción de apoyo de la persona por parte de su núcleo familiar y por parte de sus amigos (González y Landero 2014), y en estudios previos su confiabilidad ha sido 
a o,84 para la dimensión de apoyo social y de o,82 para la dimensión de amigos (Rodríguez y otros, 2016). En el presente estudio se reportaron medidas de confiabilidad de 0,77 para la dimensión de familia y de 0,75 para amigos.

Finalmente, para la medición de la motivación de logro se utilizó la escala de Manassero y Vásquez (1998) con la adaptación realizada por Durán-Alponte y Pujol (2013), dando como resultado la Escala de motivación al logro-General (EAML-G). Esta escala está conformada por 18 reactivos de diferencial semántico y el participante debe contestar cada ítem a través de una escala que va entre 1 a 6 puntos, donde las puntuaciones más altas en cada ítem se corresponden con el sentido de la motivación más favorable para el éxito académico (Morales, 2012). Un ejemplo de ítems en esta escala sería cuál es el grado de satisfacción que tiene el estudiante en relación con sus notas del semestre anterior. Los resultados psicométricos de esta escala fueron reportados por Durán-Aponte y Pujol (2013), quienes validaron esta escala sosteniendo la adecuada estructura interna de la prueba, la cual en su conjunto dan cuenta de la motivación de logro del estudiante. Las autoras reportan además un índice de consistencia interna a través del alfa de Cronbach de o,81 para la escala total. Para esta investigación la confiabilidad de la prueba reportó un alfa de Cronbach de o,89.

\section{Aspectos éticos}

La elaboración del diseño de investigación y posterior análisis de datos se ajustó a los criterios éticos y legales vigentes a nivel nacional e internacional con respecto a los principios fundamentales de transparencia, respeto, beneficio y confidencialidad en la investigación con seres humanos (Richaud, 2007), el cual se operacionalizó mediante el documento de consentimiento informado cuyo objetivo estaba destinado a informar a todos los probables participantes sobre los fines de la investigación, lo que se les solicita que realicen en su calidad de participantes y además se les informa sobre sus derechos de confidencialidad, anonimato y posibilidad de retirarse del estudio cuando estime conveniente. Cabe destacar que para la realización de este estudio se contó con la aprobación y visación por parte del Comité de Ética de la institución de origen al que pertenecen los afiliados a esta investigación.

\section{Procedimiento}

Se tomó contacto con las autoridades del Programa de Acompañamiento y Acceso a la Educación Superior de la casa de estudio de donde se extrajo la muestra con la finalidad de acceder al contacto con los posibles participantes. A través del correo electrónico de los estudiantes se les invitó a participar una vez finalizado el primer semestre lectivo y a comienzos del segundo semestre. A dicho correo se adjuntó un enlace mediante la plataforma Google drive $\odot$ con el consentimiento informado, 
donde debían seleccionar la opción «aceptar» en forma previa a la plataforma con los cuestionarios y preguntas sociodemográficas que se utilizaron. Adicionalmente, se les solicitó autorización para acceder a las calificaciones promedio obtenidas durante el primer semestre que había finalizado recientemente.

\section{Análisis de datos}

Para el tratamiento de los datos y presentación de las variables de estudio, se realizaron análisis descriptivos (promedios y desviaciones estándar para rasgos de personalidad, percepción de apoyo, motivación de logro y rendimiento académico - operacionalizado en función del promedio total obtenido por los estudiantes durante el primer semestre cursado en la universidad-). Posteriormente, se realizaron análisis bivariados (para evaluar las diferencias por sexo en cada una de las variables estudiadas mediante la prueba $\mathrm{T}$ student, y para establecer la relación entre estas se utilizó el coeficiente de correlación de Pearson). Previamente, se constató la normalidad de la distribución de datos mediante las puntuaciones de asimetría y curtosis, cumpliéndose el supuesto de normalidad. Finalmente, se realizó un análisis de regresión lineal múltiple con el método stepwise, considerando como variable dependiente el rendimiento académico de los estudiantes, mientras que las variables independientes que se incluyeron en el modelo fueron las que resultaron ser estadísticamente significativas en los análisis correlacionales previos. Todos los análisis se realizaron con el programa estadístico SPSS@ versión 23.

\section{Resultados}

En la tabla 1 se presenta un resumen de los estadísticos descriptivos de cada una de las variables estudiadas. Adicionalmente se presentan comparaciones de estas variables en función del sexo de los participantes, encontrándose que no existen diferencias significativas en ninguna de las variables estudiadas entre hombres y mujeres.

Para analizar las relaciones existentes entre el rendimiento académico (operacionalizado como la calificación promedio obtenida por los estudiantes durante el primer semestre cursado en la universidad) con los rasgos de personalidad, la percepción de apoyo social y la motivación de logro, el coeficiente de correlación de Pearson mostró que este se relaciona de manera significativa con la percepción de apoyo social de la familia ( $\mathrm{r}=.29 ; \mathrm{p} \leq .01)$, percepción de apoyo social de los amigos $(\mathrm{r}=.43 ; \mathrm{p} \leq .01)$, motivación de logro $(\mathrm{r}=.52 ; \mathrm{p} \leq .01)$ y con los rasgos de personalidad responsabilidad $(\mathrm{r}=.35 ; \mathrm{p} \leq .01)$ y apertura a la experiencia $(\mathrm{r}=.42 ; \mathrm{p} \leq .01)$. La tabla 2 presenta las correlaciones detalladas entre las variables. 
Tabla 1. Estadísticos descriptivos de las variables por tipo de muestra.

\begin{tabular}{|lccccccccc|}
\hline Variables de estudio & \multicolumn{2}{c}{ Global (155) } & \multicolumn{2}{c}{ Hombres $(\mathbf{n = 6 7})$} & \multicolumn{2}{c}{ Mujeres $(\mathbf{n = 8 8})$} & t & p \\
\hline & M & DS & M & DS & M & DS & & \\
Motivación escolar & 4.29 & .62 & 4.25 & .64 & 4.29 & .60 & -.417 & .677 \\
Percepción de apoyo social & & & & & & & & \\
Percepción familiar & 4.02 & .74 & 3.97 & .72 & 4.09 & .75 & -.993 & .332 \\
Percepción amigos & 4.04 & .75 & 4.00 & .74 & 4.07 & .77 & -.613 & .541 \\
Rasgos de personalidad & & & & & & & & \\
Neuroticismo & 3.08 & .64 & 2.97 & .68 & 3.16 & .62 & -1.89 & .060 \\
Apertura a la experiencia & 3.59 & .69 & 3.65 & .73 & 3.55 & .68 & .945 & .346 \\
Responsabilidad & 3.37 & .55 & 3.29 & .54 & 3.40 & .56 & -1.24 & .215 \\
Extraversión & 3.05 & .57 & 3.08 & .57 & 3.00 & .58 & .803 & .423 \\
Agradabilidad & 3.52 & .68 & 3.59 & .55 & 3.54 & .69 & .517 & .606 \\
Rendimiento académico & 4.44 & .91 & 4.46 & .96 & 4.42 & .88 & .341 & .734 \\
\hline
\end{tabular}

Fuente: Elaboración propia.

Tabla 2. Correlaciones entre las variables de rendimiento académico, motivación de logro, percepción de apoyo social y rasgos de personalidad.

\begin{tabular}{|lccccccccc|}
\hline Variables de estudio & $\mathbf{1}$ & $\mathbf{2}$ & $\mathbf{3}$ & $\mathbf{4}$ & $\mathbf{5}$ & $\mathbf{6}$ & $\mathbf{7}$ & $\mathbf{8}$ & $\mathbf{9}$ \\
\hline Motivación de logro & - & & & & & & & \\
Percepción apoyo familiar & $.37^{* *}$ & - & & & & & & \\
\hline Percepción apoyo amigos & $.51^{* *}$ & $.74^{* *}$ & - & & & & & \\
Neuroticismo & $-.18^{*}$ & -.07 & $-.18^{*}$ & - & & & & \\
Apertura a la experiencia & $.38^{* *}$ & $.30^{* *}$ & $.28^{* *}$ & -.11 & - & & & \\
Responsabilidad & $.46^{* *}$ & $.29^{* *}$ & $.38^{* *}$ & $-.17^{*}$ & $.29^{* *}$ & - & & \\
Extraversión & $.19^{*}$ & $.25^{* *}$ & .11 & $-.21^{* *}$ & $.28^{* *}$ & .08 & - & \\
Agradabilidad & .05 & $.25^{* *}$ & $.19^{*}$ & $-.18^{*}$ & $.20^{*}$ & $.32^{* *}$ & .07 & - & \\
\hline Rendimiento académico & $.52^{* *}$ & $.29^{* *}$ & $.43^{* *}$ & -.05 & $.42^{* *}$ & $.35^{* *}$ & .13 & -.01 & - \\
\hline
\end{tabular}

Fuente: Elaboración propia. 


\section{Variables personales predictoras del rendimiento académico}

Dado que el objetivo de esta investigación era establecer de qué manera las variables personales motivación de logro, percepción de apoyo social y rasgos de personalidad eran predictoras del rendimiento académico, se realizó un análisis de regresión lineal múltiple que permite esclarecer con mayor precisión la red de interrelaciones que se establecen entre las variables seleccionadas como predictoras con la variable criterio. Para esto, primeramente se comprobaron los supuestos básicos requeridos para realizar este tipo de análisis de regresión, en el cual se da por satisfecho el criterio de independencia de errores, normalidad, linealidad, homocestacidad y multicolinealidad.

Una vez realizado este procedimiento con el método stepwise se fueron incluyendo en el modelo de regresión propuesto una a una las variables que mayor fuerza y significancia resultaron tener en la correlación con el rendimiento académico (ver tabla 2). Así, el modelo resultante final incluyó la motivación de logro, la percepción de apoyo de la familia y la percepción de apoyo de los amigos, como también los rasgos de personalidad, responsabilidad y apertura a la experiencia como variables predictoras importantes del rendimiento académico de los estudiantes. De esta forma, como se ve en la tabla 3, el modelo propuesto explica el 33,3\% de la varianza total del rendimiento académico $(\mathrm{F}=15.76 ; \mathrm{gl}=5,143 ; \mathrm{p}=.000)$.

Tabla 3. Variables personales predictoras del rendimiento académico.

\begin{tabular}{|c|c|c|c|c|c|c|c|}
\hline \multirow[b]{2}{*}{ Predictores } & \multicolumn{2}{|c|}{ Datos predictores } & \multicolumn{5}{|c|}{ Datos modelo } \\
\hline & $\beta$ & $\mathrm{t}$ & $\mathrm{p}$ & $95 \%$ IC $\beta$ & R2 & R2 Ajust. & $\mathrm{F}$ \\
\hline Motivivación. Logro & .299 & 3.37 & .001 & $.183-.705$ & .355 & .333 & 15.76 \\
\hline Percepción apoyo social y amigos & .272 & 2.40 & .018 & $.058-.602$ & & & \\
\hline Apertura experiencia & .255 & 3.40 & .001 & $.140-.527$ & & & \\
\hline Responsabilidad & .064 & .827 & .410 & $-.150-.365$ & & & \\
\hline Percepción apoyo familiar & -.151 & -.455 & .148 & $-.440-.067$ & & & \\
\hline
\end{tabular}

Fuente: Elaboración propia.

Al evaluar a través del coeficiente $\beta$ las magnitudes del efecto directo de cada una de las variables por separado sobre el rendimiento académico, se encontró que la variable que más aporta a la predicción es motivación con una correlación positiva y significativa $(\beta=.299, \mathrm{p}=.001)$ seguida de percepción de apoyo social amigos $(\beta=.272$, $\mathrm{p}=.018)$ y el rasgo de personalidad apertura a la experiencia $(\beta=.255, \mathrm{p}=.001)$. Las restantes variables no fueron significativas.

Finalmente, cabe advertir que al no encontrarse diferencias significativas en las variables de estudio entre hombres y mujeres, se realizó este análisis de regresión en conjunto sin contemplar una separación por sexo. 


\section{Conclusión}

Explicar el rendimiento académico de estudiantes universitarios a partir de variables psicológicas resulta un factor clave para la toma de decisiones en materia de intervención en el ámbito de la inclusión universitaria, y de forma particular cuando se piensa en los sectores vulnerables. Para Barahona (2014), este rendimiento académico debe ser comprendido de forma multifactorial y no como un hecho unidireccional o lineal, debido a su carácter complejo donde intervienen variables de orden psicológicas (cognitivas, emocionales, motivacionales, de autoeficacia), sociales e institucionales (Himmel, 2002; González, 2005; Garbanzo, 2007), los que permiten contextualizarlo y así favorecer un análisis más cercano a los y las estudiantes.

En los resultados hemos apreciado que las variables que se asocian más fuertemente al rendimiento académico son la motivación (de logro), la percepción de apoyo de pares y la apertura a la experiencia como factor de personalidad, constituyéndose en buenos predictores de la permanencia universitaria de estudiantes vulnerables a partir de este tipo de variables académicas.

En el caso de la motivación, esta actúa como una variable significativa y de importancia en el modelo. Los datos son consistentes con gran parte de la literatura asociada, reforzando teóricamente su peso predictivo en el desempeño académico en general. Esto es interesante debido a las siguientes razones: i) la motivación es un factor que se asocia significativamente con el rendimiento académico (Reed, 1968); ii) la motivación está principalmente relacionada con el tipo de deserción temporal a diferencia de otros factores que se asocian principalmente con la de tipo permanente (Canales y De Los Ríos, 2007); y iii) el objetivo de las orientaciones técnicas generales de implementación de un Programa de Acompañamiento y Acceso a la Educación Superior en educación media y de acompañamiento en la permanencia universitaria, involucran a la motivación y los intereses personales de los y las estudiantes que participan en él, aportando así a la configuración de un itinerario educativo postsecundario, universitario y/o laboral que influya en sus decisiones.

Con respecto a la percepción de apoyo social, esta se aprecia como un buen predictor del rendimiento académico en estos estudiantes. Esto es consistente con el concepto de apoyo social presente en autores como Domínguez y otros (2011), quienes han señalado que el estudiante que se enfrenta a una situación estresante considera que no tan solo requiere de sus propios recursos para afrontarla, sino que también recurre a otros pares de su red cercana (amistad), de manera que dicho afrontamiento sea menos demandante y más fácil. En el caso de los estudiantes del Programa de Acompañamiento y Acceso a la Educación Superior, resulta fundamental profundizar en la comprensión del proceso de inclusión académico-social al sistema universitario, asunto de vital importancia, ya que el y la estudiante no solo se deben al rendimiento asociado a los contenidos curriculares específicos, sino también a la vin- 
culación que consigue en un contexto de apoyo y beneficio tanto con la institución como con sus pares (Tinto, 1989). La identificación y aceptación del entorno social actúa como un apoyo altamente significativo en coherencia con lo señalado anteriormente, actuando como red de acompañamiento emocional, académico y social frente a las altas dificultades académicas percibidas como aversivas debido a las condiciones de vulneración escolar y curricular de origen.

La apertura a la experiencia se presenta como un rasgo de personalidad significativo que cobra un particular interés en este contexto, con un buen peso predictivo del rendimiento académico. Se observa relacionado con la motivación y, en forma más fuerte, con la percepción de apoyo de la red cercana, lo que es consistente con lo señalado en los modelos de retención universitaria que aluden a la importancia institucional de la universidad como espacio educativo donde no tan solo se requieren conocimientos curriculares específicos, sino donde se vinculan acciones de extensión que permitan descubrir nuevas perspectivas, aportar nuevas discusiones y experimentar nuevos desafíos creativos personales. Asimismo, autores como Román y Hernández (2005) y Martín (2007) han señalado respectivamente que el apoyo social fomenta la obtención de buenos resultados académicos, así como una más efectiva forma de afrontar los estresores académicos.

Para Gallardo y otros (2014), el estudiante vulnerable transitaría en su inclusión universitaria por cuatro procesos identificables, ${ }^{3}$ destacándose el denominado «período de extrañeza», donde la apertura a la experiencia cobra importancia fundamental, estableciendo las bases iniciales para posibilitar un buen proceso de inserción y fortaleciendo al estudiante en el ámbito social, apoyándose en otros estudiantes en situación similar (en el caso del Programa de Acompañamiento y Acceso a la Educación Superior). En este sentido, el y la estudiante poseen una característica esencial para romper con la amenaza de estereotipo, y avanzar en su adaptación a un contexto más allá de sus condiciones vulneradas de origen escolar, social y familiar (Furrer, 2013).

En relación con la responsabilidad, esta se asocia con un aumento en el rendimiento académico de los y las estudiantes, inclusive permitiendo prever resultados exitosos. Esto ha sido confirmado recientemente en un estudio con universitarios del norte de Chile, donde se comprobó que la responsabilidad es el mejor predictor del rendimiento en relación con resto de las dimensiones del Big Five Inventory (Cuadra y otros, 2015). Sin embargo, en este estudio no se corrobora dicha afirmación, apreciándose que si bien existe una relación significativa entre ambas variables (responsabilidad y rendimiento), esta no actúa como un predictor suficiente del rendimiento académico. En este sentido, es pertinente preguntarse qué significa ser responsable para estos estudiantes. Al existir en este estudio una significativa correlación entre

3. Los cuatro factores descritos son: selección de la institución; período de inducción; período de extrañeza; y período de evaluación y continuidad. 
esta y la apertura a la experiencia, es interesante profundizar acerca de estos indicadores, considerando que asumir una posición responsable no precisamente se ajusta al cumplimiento de requerimientos académicos excluyentes, sino también a cómo los y las estudiantes van entendiendo dicha responsabilidad como la exploración de un nuevo contexto donde se presentan desafíos y nuevas lógicas culturales, y cómo esto eventualmente puede afectar en la decisión de permanencia. Lo anterior es coherente con la integración académico-social señalada por Tinto $(1975,1989)$, aludiendo la importancia de comprender que no tan solo de factores económicos y académicos depende la inclusión, sino también de cómo los estudiantes se comprometen con la institución y las posibilidades que de esta aprovechan.

Como limitaciones del estudio se mencionan que es de tipo transversal y no establece un proceso de seguimiento de los estudiantes, entendiendo que la motivación, el apoyo de pares y la vivencia de la experiencia van transitando en forma dinámica a lo largo de un semestre, por lo que la predicción que genera el modelo debe ser interpretada con cautela y rigor en la evidencia empírica y teórica. Otra limitación la constituye el tipo de muestra, que no permite generalizar a toda la población de estudiantes del Programa de Acompañamiento y Acceso a la Educación Superior, por lo que se hace necesario ampliar los estudios hacia muestras representativas, con diseño de carácter longitudinal, que reflejen en forma más fiel el proceso y la forma en que estas variables en la población se manifiestan.

En síntesis, los resultados sugieren que desde la perspectiva psicológica los estudiantes deben ser considerados en sus motivaciones, intereses y proyecciones, asuntos que en su definición asumen las orientaciones actuales de los programas de acompañamiento y acceso a la educación superior, planteando directrices en el camino correcto, y entendiendo a los y las estudiantes en forma multifacética. Sin duda que estos factores psicológicos asociados a los de la cultura escolar, pedagógicos, curriculares y sociales-familiares, permitirán orientar nuevos procesos de intervención y desarrollo de los estudiantes a partir de su educación secundaria, con miras hacia la tan anhelada inclusión en equidad de los estudiantes que históricamente han sido vulnerados desde la perspectiva educacional en su derecho para el acceso y permanencia en la enseñanza universitaria.

\section{Financiamiento}

Esta investigación fue financiada por la Dirección de Investigación Científica y Tecnológica (DICYT) de la Universidad de Santiago de Chile (Proyecto 031893MN). 


\section{Referencias}

Aто, Manuel, Juan López, y Ana Benavente (2013). «Un sistema de clasificación de los diseños de investigación en psicología». Anales de Psicología, 29 (3): 1038-1059. DOI: 10.6018/analesps.29.3.178511.

Barahona, Plank (2014). «Factores determinantes del rendimiento académico de los estudiantes de la Universidad de Atacama». Estudios Pedagógicos, 40 (1): 25-39. DOI: $10.4067 /$ So718-07052014000100002.

BISQUERRA, Ricardo (2016). «Educación emocional». Conferencia pronunciada en I Jornadas del máster en resolución de conflictos en el aula. Valencia: Máster de resolución de conflictos en el aula.

Benet-Martínez, Verónica y John Oliver (1998). «Los Cinco Grandes Across Cultures and Ethnic Groups: Multitrait Multimethod Analyses of the Big Five in Spanish and English». Journal of Personality and Social Psychology, 75 (3): 729-750. DOI: 10.1037//0022-3514.75.3.729.

Canales, Andrea y Danae De los Ríos (2009). «Retención de estudiantes vulnerables en la educación universitaria chilena». Revista Calidad en la Educación, 30: 50-83. DOI: 10.31619/caledu.n30.173.

Canales, Andrea y Danae De los Ríos (2007). «Factores explicativos de la deserción universitaria». Revista Calidad en la Educación, 26: 173-201. DOI: 10.31619/caledu. n26.239.

Castro, Juan, Claudio Frites y Pablo Vargas (2016). «El Programa de Acompañamiento y Acceso Efectivo a la Educación Superior (PACE)». Estudios de Caso, 135: 1-12. Disponible en https://bit.ly/2ELVM9Z.

Cuadra, Alejandro, Constanza Veloso, Karina Marambio y Carolina Tapia (2015). «Relación entre rasgos de personalidad y rendimiento académico en estudiantes universitarios». Interciencia, 40 (10): 690-695. Disponible en https://bit. ly/2Zq6qgk.

De Hoyos, Rafael, Juan Espino y Vicente García (2010). Determinantes del logro escolar en México: primeros resultados utilizando la prueba ENLACE media superior. Ciudad de México: Secretaría de Educación Media Superior.

Domínguez, Alejandra, Irene Salas, Carolina Contreras y Mary Procidano (2011). «Validez concurrente de la versión mexicana de las escalas de apoyo social percibido de la familia y los amigos (PSS-Fa y PSS-Fr)». Revista Latinoamericana de Psicología, 43 (1): 125-137. Disponible en https://bit.ly/2ZkR7Fu.

Donoso, Cristian y Ernesto Schiefelbein (2007). «Análisis de los modelos explicativos de retención de estudiantes en la universidad: una visión desde la desigualdad social». Estudios pedagógicos, 33 (1): 7-27. DOI: 10.4067/S0718-07052007000100001. Dubet, François (2004). La escuela de las oportunidades. Barcelona: Gedisa.

Durán-Aponte, Emilse y Lydia Pujol (2013). «Escala Atribucional de Motivación de 
Logro General (EAML-G): Adaptación y análisis de sus propiedades psicométricas». Estudios Pedagógicos, 39 (1): 83-97. DOI: 10.4067/So718-07052013000100005. EDEL, Rubén (2003). «El rendimiento académico: concepto, investigación y desarrollo». Revista Electrónica Iberoamericana sobre Calidad, Eficacia y Cambio en Educación (REICE), 1 (2): 1-15.

ELLIOT, Colin (1972). «Personality factors and scholastic attainment». British Journal of Educational Psychology, 42 (1): 23-32. DOI: 10.1111/j.2044-8279.1972.tboo691.x.

Escudero, Aura (2015). «Evaluación del área facilitadores del programa de preparación y acceso efectivo a la educación superior (PACE)». Tesis de Magíster. Santiago: Pontificia Universidad Católica de Chile. Disponible en https://bit.ly/2s82Dbm.

EspinOzA, Óscar, Dante Castillo, Luis González y Javier Loyola (2012). «Factores familiares asociados a la deserción escolar en Chile». Revista de Ciencias Sociales, 18 (1): 136-150. DOI: 10.4067/S0718-07052014000100006.

Fernández-González, Luis, Antonio González-Hernández y María Victoria Trianes-Torres (2015). «Relaciones entre estrés académico, apoyo social, optimismo-pesimismo y autoestima en estudiantes universitarios». Electronic Journal of Research in Educational Psychology, 13 (1): 111-130. Disponible en https://bit. ly/2ENveFs.

FURrer, Sofía (2013). «Comprendiendo la amenaza de estereotipo». Revista electrónica de investigación docencia creativa, 2: 239-260. Disponible en https://bit. ly/2SrvgLo.

Gaxiola, José, Sandybell González, Zita Contreras y Eunice Gaxiola (2012). «Predictores del rendimiento académico en adolescentes con disposiciones resilientes y no resilientes». Revista de Psicología, 30 (1): 47-74. Disponible en https://bit. ly/2ZjYWeP.

GarbanZo, Giselle (2007). «Factores asociados al rendimiento académico en estudiantes universitarios, una reflexión desde la calidad de la educación superior pública». Revista Educación, 31 (1): 43-63. Disponible en https://bit.ly/2ZiJTBN.

GiL, Francisco, Ricardo Paredes e Ignacio Sánchez (2013). «El ranking de las notas: inclusión con excelencia». Centro de políticas públicas UC, 8 (60): 3-19. Disponible en https://bit.ly/2seKzMC.

Goldthorpe, John (2012). «Back to Class and Status: Or Why a Sociological View of Social Inequality Should Be Reasserted». Reis 137: 201-216. DOI: 10.5477/cis/ reis.137.201.

Golovanevsky, Laura (2007). «Vulnerabilidad Social: Una Propuesta para su Medición en Argentina». Revista de economía y estadística, 14 (2): 53-94. Disponible en https://bit.ly/2sZH9xq.

GonzÁLEZ, Luis (2005). «Estudio sobre la repitencia y deserción en la educación superior chilena». Unesco: Digital Observatory for higher education in Latin America and The Caribbean. 
GonZÁLEZ, Mónica y René Landero (2014). «Propiedades psicométricas de la escala de Apoyo Social Familiar y de Amigos (AFA-R) en una muestra de estudiantes». Acta de Investigación Psicológica, 4 (2): 1469-1480. DOI: 10.1016/S2007-4719(14)70387-4.

GuZmán, Eduardo (2018). «Entre lo académico y lo emocional en la cultura escolar, el profesor en contrapunto». En Formação psicosocial na América Latina, VII Congresso da Alfepsi (pp. 66-74). Porto Alegre. Alfepsi.

Himmel, Erika (2002). «Modelos de análisis para la deserción estudiantil en la educación superior». Calidad de la Educación, 17: 91-107. DOI: 10.31619/caledu.n17.409.

Infante, Marta, Claudia Matus, Abraham Paulsen, Alejandro Salazar y Ruby Vizcarra (2012). «Narrando la vulnerabilidad escolar: performatividad, espacio y territorio». Literatura y Lingüistica, 27: 281-308. Disponible en https://bit.ly/2PSxxo4.

JADUE, Gladys (2002). «Factores psicológicos que predisponen al bajo rendimiento, al fracaso y a la deserción escolar». Estudios Pedagógicos, 28: 193-204. DOI: 10.4067/So718-07052002000100012.

John, Oliver, Eric Donahue y Robert Kentle (1991). The Big-Five Inventory-Version $4 a$ and 54. Berkeley: Berkeley Institute of Personality and Social Research.

JimÉNEZ, Manuel (2000). «Competencia social: intervención preventiva en la escuela». Infancia y Sociedad, 24: 21-48. Disponible en https://bit.ly/2EPcp4A.

Manassero, María Antonia, y Ángel Vázquez (1998). «Validación de una escala de motivación de logro». Psicothema, 10 (2): 333-351. Disponible en https://bit. ly/2PR3nL2.

Martín, Isabel (2007). «Estrés académico en estudiantes universitarios». Apuntes de Psicología, 25 (1): 87-99. Disponible en https://bit.ly/398NSWk.

Martínez, José (2014). «Clase obrera, género y éxito educativo: inteligencia, expectativas y didáctica». Rase, 7 (2): 449-467. Disponible en https://bit.ly/2PQ2 PEX.

Mineduc (2018). «Orientaciones técnicas para los componentes de preparación en la educación media y de acompañamiento en la educación superior». Santiago: Ministerio de Educación.

-. (2008). "Marco para la buena enseñanza». Santiago: Ministerio de Educación, Centro de Perfeccionamiento, Experimentación e investigaciones pedagógicas.

Morales, Samuel (2012). «Estudio del nivel de empatía y motivación de logro de los alumnos de la carrera de Odontología de la Universidad de Concepción». Revista de Educación en Ciencias de la Salud, 9 (2): 121-125. Disponible en https://bit. ly/399obVm.

Morales-Vallejo, Pedro (2008). Estadística aplicada a las ciencias sociales. Madrid: Universidad Pontificia Comillas.

Morales, Mario (2014). "Características intelectuales, psicoemocionales y afectivas de estudiantes de alto rendimiento pertenecientes a liceos prioritarios». Revista de Pedagogía Universitaria y Didáctica del Derecho, 1 (2): 48-56. DOI: 10.5354/0719-5885.2015.3598. 
Morales, Mario y Eduardo Guzmán (2016). «Vivencias vitales significativas, bienestar psicológico y resiliencia de estudiantes de alto rendimiento escolar pertenecientes a sectores vulnerables». Revista de Investigación en Psicología, 19 (2): 25-47. DOI: 10.15381/rinvp.v19i2.12888.

Morales, Mario y Adolfo González (2014). «Resiliencia-autoestima-bienestar psicológico y capacidad intelectual de estudiantes de cuarto medio de buen rendimiento de liceos vulnerables». Estudios Pedagógicos, 11 (1): 215-228. DOI: 10.4067/ So718-07052014000100013.

NiÑo, Isabel, Arturo Calderón y Mónica Cassaretto (2003). «Personalidad y rendimiento académico en estudiantes universitarios». Revista de Psicología (PUCP), 21 (1): 120-143.

NovoA, Consuelo y Enrique Barra (2015). «Influencia del apoyo social percibido y los factores de personalidad en la satisfacción vital de estudiantes universitarios». Terapia Psicológica, 33 (3): 239-245. DOI: 10.4067/So718-48082015000300007.

Ñanculeo, Marco. (2014). "Construcción de un índice de vulnerabilidad social para estudiantes preescolares de establecimientos subvencionados de Chile». Tesis de Magíster. Concepción: Universidad de Concepción. Disponible en https://bit. ly/2SoZqP6.

O'Connor, Melissa y Sampo Paunonen (2007). «Big Five personality predictors of post-secondary academic performance». Personality and Individual Differences, 43: 971-990. DOI: 10.1016/j.paid.2007.03.017.

Poropat, Arthur (2009). «A meta-analysis of the five-factor model of personality and academic performance». Psychological Bulletin, 135 (2): 322-338. DOI: 10.1037/ aoo14996.

RAHMER, Beatriz, Rafael Miranda y Francisco Gil (2013). «Programa de Acceso Inclusivo Equidad y Permanencia de la Universidad de Santiago de Chile: Una Política de Acción Afirmativa». Ponencia pronunciada en la Conferencia Latinoamericana sobre el Abandono en la Educación Superior. Ciudad de México: Universidad Autónoma de México.

Reed, Horace (1968). «College Students' Motivations Related to Voluntary Dropout and Under-Overachievement». Journal of Educational Research, 61 (9): 412-416. Disponible en https://bit.ly/2sXAxiK.

ReImers, Fernando y Connie Chung (2016). Enseñanza y aprendizaje en el siglo XXI. Metas, políticas educativas y currículo en seis países. Ciudad de México: Fondo de Cultura Económica.

RichaUd, María Cristina (2007). «La ética en la investigación psicológica». Enfoques 19 (1): 5-18. Disponible en https://bit.ly/34RKAU3.

Rinaudo, María Cristina, María Laura Barrera y Danilo Donolo (2006). «Motivación para el aprendizaje en alumnos universitarios». Revista Electrónica de Motivación y Emoción, 9 (22): 2-19. Disponible en https://bit.ly/36VgvnY. 
Rodríguez, Arantzazú, Estibaliz Ramos, Iker Ros, Arantza Fernández y Lorena Revuelta (2016). «Bienestar subjetivo en la adolescencia: el papel de la resiliencia, el autoconcepto y el apoyo social percibido». Suma Psicológica, 23, (1): 60-69. DOI: 10.1016/j.sumpsi.2016.02.002.

Román, Carlos y Yenima Hernández (2005). «Variables psicosociales y su relación con el desempeño académico de estudiantes de primer año de la Escuela Latinoamericana de Medicina». Revista Iberoamericana de Educación, 37 (2): 1-8. DOI: $10.35362 /$ rie3722718.

Schmitт, David, Jüri Allik, Robert R. McCrae y Veronica Benet (2007). «The geographic distribution of Big Five personality traits: Patterns and profiles of human self-description across 56 nations». Journal of Cross-Cultural Psychology 38: 173212. DOI: $10.1177 / 0022022106297299$.

Tinto, Vincent (1989). «Definir la deserción: una cuestión de perspectiva». Revista de Educación Superior Anuies, 71: 1-9. Disponible en https://bit.ly/2tEfSRc.

TinTo, Vincent (1975). «Dropout from higher education: A theoretical synthesis of recent research». Review of Educational Research 43, (1): 89-125. DOI: 10.3102/00346543045001089.

ToIrkens, Josselinne y Sonia Osses (2016). «Estudiantes talentosos de contextos vulnerables y su ingreso a carreras de Medicina». Revista Médica de Chile, 144 (4): 534-543. DOI: 10.4067/So034-98872016000400018.

Torres, Laura y Norma Rodríguez (2006). «Rendimiento académico y contexto familiar en estudiantes universitarios». Enseñanza e Investigación en Psicología, 11 (2): 255-270. Disponible en https://bit.ly/2PSXNI2.

Torres-Acosta, Noel, Jaime Rodríguez-Gómez y Miriam Acosta-Vargas (2013). «Personalidad, aprendizaje y rendimiento académico en medicina». Investigación en Educación Médica, 2 (8): 193-201. DOI: 10.1016/S2007-5057(13)72712-1.

\section{Sobre los autores}

Mario Morales Navarro es doctor en Ciencias de la Educación. Es académico de la Facultad de Humanidades, Escuela de Psicología, Universidad de Santiago de Chile. Su correo electrónico es mario.morales@usach.cl. (D) http://orcid. org/0000-003-4995-4414.

Eduardo Guzmán Utreras es doctor en Psicología. Es académico de la Facultad de Ciencias Sociales y Comunicaciones, Escuela de Psicología, Universidad Santo Tomás, Santiago. Su correo electrónico es eduardoguzmanut@santotomas.cl. (D) http:// orcid.org/o0oo-0002-3336-3524.

Carmen Gloria Baeza Ugarte es doctora en Psicología. Es académica de la Facultad de Humanidades, Escuela de Psicología, Universidad de Santiago de Chile. Su correo electrónico es carmen.baeza@usach.cl. (D) http://orcid.org/oooo-ooo1-5316-3397. 
La Revista Pedagogía Universitaria y Didáctica del Derecho (RPUDD) es una publicación científica semestral que contribuye a la reflexión multidisciplinaria sobre pedagogía universitaria y didáctica del derecho, para la formación y consolidación de esta área de investigación; así como a la difusión de prácticas innovadoras en la enseñanza-aprendizaje del derecho considerando el contexto nacional e internacional. Es una publicación electrónica internacional con una codirección entre Brasil y Chile.

\author{
DIRECTORA \\ María Francisca Elgueta Rosas \\ Universidad de Chile \\ DIRECTOR \\ Renato Duro Dias \\ Universidad Federal de Rio Grande, Brasil \\ SITIO WEB \\ pedagogiaderecho.uchile.cl \\ CORREO ELECTRÓNICO \\ rpedagogia@derecho.uchile.cl \\ LICENCIA DE ESTE ARTÍ́CULO
}

Creative Commons Atribución Compartir Igual 4.0 Internacional

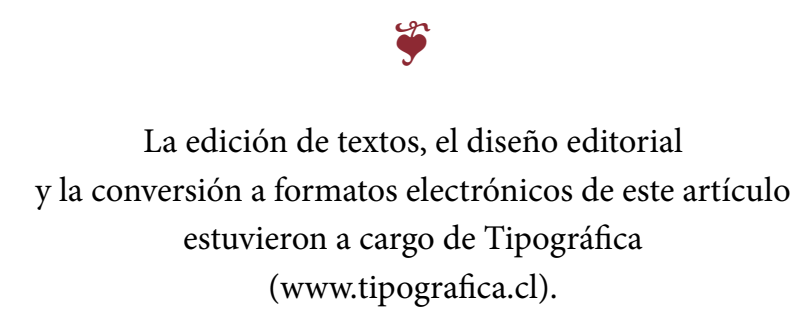

